Europhysics Letters

PREPRINT

\title{
Wetting at Curved Substrates: Non-Analytic Behavior of Interfacial Properties
}

\author{
R. Evans ${ }^{1,2,3}$, R. Roth ${ }^{2,3}$ and P. BRYK ${ }^{2,3,4}$ \\ 1 H.H. Wills Physics Laboratory, University of Bristol, Bristol BS8 1TL, UK \\ 2 Max-Planck-Institut für Metallforschung, Heisenbergstr. 3, 70569 Stuttgart, Germany \\ 3 ITAP, Universität Stuttgart, Pfaffenwaldring 57, 70569 Stuttgart, Germany \\ 4 Dep. of Modeling of Physico-Chemical Processes, MCS University, Lublin 20-031, \\ Poland
}

PACS. 05.70.Np - Interface and surface thermodynamics.

PACS. 68.08.Bc - Wetting.

\begin{abstract}
We argue that for complete wetting at a curved substrate (wall) the wall-fluid surface tension is non-analytic in $R_{i}^{-1}$, the curvature of the wall and that the density profile of the fluid near the wall acquires a contribution proportional to the gas-liquid surface tension $\times R_{i}^{-1}$ plus higher-order contributions which are non-analytic in $R_{i}^{-1}$. These predictions are confirmed by results of density functional calculations for the square-well model of a liquid adsorbed on a hard sphere and on a hard cylinder where complete wetting by gas (drying) occurs. The implications of our results for the solvation of big solvophobic particles are discussed.
\end{abstract}

Understanding the adsorption of fluids at solid substrates has taken on new importance with recent advances in the controlled fabrication of tailored surfaces for applications in microfluidics and other areas [1]. Much experimental [2] and theoretical effort [3] is concerned with wetting and associated interfacial transitions in wedge geometry and recently attention has turned to wetting at an apex [4. It is becoming increasingly clear that substrate geometry can have a profound influence on the nature of fluid adsorption and, in particular, on wetting characteristics making these quite different from those at a planar substrate. Here we consider complete wetting at two substrates that have simple geometries, namely a single sphere of radius $R_{s}$ and an infinitely long cylinder of radius $R_{c}$. Using an effective interfacial Hamiltonian approach [5] combined with exact microscopic sum-rules for the density profile of the fluid near a hard wall, we show that in the limit $R_{i} \rightarrow \infty$, with $i=s, c$, the surface tension of the substrate (wall)-fluid interface is non-analytic in the curvature $R_{i}^{-1}$ and that the density of the fluid in contact with the hard wall acquires a contribution proportional to $\gamma_{g l}(\infty) / R_{i}$, where $\gamma_{g l}(\infty)$ is the surface tension of the planar interface between coexisting gas and liquid, as well as higher-order non-analytic terms in $R_{i}^{-1}$. Our results, which are confirmed fully by the results of microscopic density functional (DFT) 6 calculations, show that non-zero curvature leads to unexpected and subtle effects on interfacial properties when complete wetting occurs, even for the simplest of substrate geometries.

In previous theoretical studies of wetting on spheres and cylinders [ 789 10] the thrust was on understanding how a finite radius limits the thickness of a wetting film and modifies the

(c) EDP Sciences 
wetting transition that can occur at a planar substrate. Little attention was paid to the effect of curvature on the surface tension and on the form of the density profile near the substrate which are the main concerns of this Letter. Our results have repercussions for the general theory of solvation of big solvophobic solute particles, for wetting of colloidal particles and fibers [1] 10] and, possibly, for the surface tension of drops and bubbles [11.

We consider first the general case of a bulk fluid phase $a$, at chemical potential $\mu$, in contact with a wall $w$ and implement a standard, coarse-grained, effective interfacial Hamiltonian approach [5, 8] in which the wetting film of fluid phase $b$ is characterized only by its thickness $l$. For short-ranged (finite ranged, exponentially or faster decaying) wall-fluid and fluid-fluid potentials the binding potential, i.e. the excess (over bulk) grand potential per unit area, takes the form [5, 8 :

$$
\tilde{\omega}_{w a}^{i}(l)=\gamma_{w b}^{i}\left(R_{i}\right)+\gamma_{b a}^{i}\left(R_{i}+l\right)+\tilde{a}(T) e^{-l / \xi}+\left(\rho_{a}-\rho_{b}\right) \delta \mu l+\frac{\alpha_{i} \gamma_{b a}^{i}\left(R_{i}+l\right) l}{R_{i}}+\mathcal{O}\left(l / R_{i}\right)^{2},
$$

with $i=s, c$ and $\alpha_{s}=2, \alpha_{c}=1$ for the spheres and cylinders, respectively. $\gamma_{w b}^{i}$ is the surface tension of the wall-phase $b$ interface, $\gamma_{b a}^{i}$ is the tension of the $b a$ fluid-fluid interface located near $R_{i}+l, \tilde{a}(T)>0$ is a coefficient that we need not specify further for complete wetting, and $\xi$ is the true bulk correlation length of the wetting phase $b$. The fourth term in eq. (ID) accounts for the increase in grand potential per unit area associated with the volume of a film of phase $b ; \delta \mu \equiv \mu-\mu_{c o}(T)$ is the chemical potential deviation and $\rho_{a}, \rho_{b}$ are the number densities of bulk phases $a$ and $b$ at coexistence $\mu_{c o}(T)$. For $R_{i}=\infty$ the binding potential (11) reduces to that appropriate for complete wetting at a planar interface in models where the range of the wall-fluid potential is shorter than $\xi$ and, henceforward, we shall assume this is the case. Then minimizing eq. (1) w.r.t. $l$ yields the well-known logarithmic divergence of the equilibrium film thickness: $l_{e q}(\infty)=-\xi \ln \left(\delta \mu \xi\left(\rho_{a}-\rho_{b}\right) / \tilde{a}(T)\right)$, as $\delta \mu \rightarrow 0$. When $R_{i} \neq \infty$ two important modifications arise: i) $\gamma_{w b}^{i}$ and $\gamma_{b a}^{i}$ now depend on curvature and ii) the surface area of the $b a$ interface now depends on the film thickness $l$. Equation (1) assumes very large radii $R_{i}$ and that $l / R_{i} \ll 1$. Note that the fifth term in (11) is proportional to the Laplace pressure across the fluid-fluid $b a$ interface. Since $\gamma_{b a}$ is always non-zero, away from the bulk critical point, this term ensures that the film thickness remains finite even at $\mu_{c o}(T)$, i.e. minimizing eq. (11) yields $l_{e q}^{i}\left(R_{i}\right)=-\xi \ln \left[\alpha_{i} \xi \gamma_{b a}^{i}\left(R_{i}\right) /\left(R_{i} \tilde{a}(T)\right)\right]$ for $\delta \mu=0$, where we have ignored terms $\mathcal{O}\left(l / R_{i}\right)^{2}$ and the $l$ dependence in $\gamma_{b a}^{i}$ - this can be justified a posteriori. Several authors [7, 8, 9, 10, have argued that for large $R_{i}$ the quantity $\alpha_{i} \gamma_{b a}^{i}\left(R_{i}\right) / R_{i}$ should play the same role in complete wetting at a curved wall, with $\delta \mu=0$, as the effective bulk field $\left(\rho_{a}-\rho_{b}\right) \delta \mu$ plays for the planar wall. We shall pursue this argument further in the present Letter.

The wall-fluid surface tension is given by

$$
\gamma_{w a}^{i}\left(R_{i}\right) \equiv \tilde{\omega}_{w a}^{i}\left(l_{e q}\right)=\gamma_{w b}^{i}\left(R_{i}\right)+\gamma_{b a}^{i}\left(R_{i}\right)+\left(\xi+l_{e q}\right)\left(\frac{\alpha_{i} \gamma_{b a}^{i}\left(R_{i}\right)}{R_{i}}+\left(\rho_{a}-\rho_{b}\right) \delta \mu\right) .
$$

For the planar interface the final term vanishes at $\delta \mu=0$ and the wall-fluid tension reduces to the sum of the planar tensions: $\gamma_{w a}(\infty)=\gamma_{w b}(\infty)+\gamma_{b a}(\infty)$, appropriate to complete wetting by a macroscopic film of phase $b$. When $\delta \mu \neq 0, \gamma_{w a}(\infty)$ acquires a non-analytic contribution proportional to $-|\delta \mu| \ln |\delta \mu|$ [5]. For finite $R_{i}$ we set $\delta \mu=0$ and obtain

$$
\gamma_{w a}^{i}\left(R_{i}\right)=\gamma_{w b}^{i}\left(R_{i}\right)+\gamma_{b a}^{i}\left(R_{i}\right)\left(1+\frac{\alpha_{i} \xi}{R_{i}}\right)+\frac{\alpha_{i} \xi \gamma_{b a}^{i}\left(R_{i}\right)}{R_{i}} \ln \left(R_{i} \times c o n s t\right)+\text { H.O.T.. }
$$


That the surface tension contains a contribution which is non-analytic in the curvature is clearly a direct manifestation of complete wetting. However, the necessity for such a contribution does not seem to have been widely recognized. An exception is Ref. [] but in that paper the consequences were not discussed. In order to elucidate these we must examine the other terms in eq. (3). At the non-wet $w b$ interface we do not expect non-analyticities in $\gamma_{w b}^{i}\left(R_{i}\right)$, provided the system exhibits short-ranged forces [12. For a spherical fluid-fluid interface it is usually assumed [1] (for short-ranged forces) that

$$
\gamma_{b a}^{s}\left(R_{s}\right)=\gamma_{b a}(\infty)\left(1-2 \delta_{T}^{s} / R_{s}+\text { H.O.T. }\right)
$$

where $\delta_{T}^{s}$ is the Tolman length 13 familiar in studies of liquid drops, whereas for cylindrical interfaces it has been argued [14 that

$$
\gamma_{b a}^{c}\left(R_{c}\right)=\gamma_{b a}(\infty)\left(1+b_{H} \ln R_{c} / R_{c}+H . O . T .\right)
$$

If the latter form were correct eq. (3) would imply that the wall-fluid tension $\gamma_{w a}^{c}\left(R_{c}\right)$ should have a contribution $\gamma_{b a}(\infty)\left(b_{H}+\xi\right) \ln \left(R_{c}\right) / R_{c}$. Provided the length $b_{H}$ is comparable with the bulk correlation length $\xi$, as is expected on physical grounds, the term in $b_{H}$ should be easily identified in numerical work.

The result (3) is valid for any complete wetting situation, with the proviso that the forces are short-ranged. We specialize now to the case of a hard wall exerting a purely repulsive potential on the fluid: $V_{i}(r)=\infty$ for radius $r<R_{i}$ and is zero for $r>R_{i}$. It is well-known that the phenomenon of complete drying occurs at a planar hard wall: the interface between the bulk liquid $(l)$ and the wall is wet by a macroscopic film of gas $(g)$ as $\mu \rightarrow \mu_{c o}^{+}(T)$ for all temperatures $T$ at which gas and liquid coexist 15]16. At $\mu=\mu_{c o}^{+}(T)$ the density profile of the fluid is a composite of the planar wall-gas and the (free) gas-liquid interfacial profiles and $\gamma_{w l}(\infty)=\gamma_{w g}(\infty)+\gamma_{g l}(\infty)$; phase $a \equiv l$ and phase $b \equiv g$. Whilst drying at a planar hard wall has been investigated extensively using computer simulations [15] and DFT [16], we are not aware of studies on curved substrates. We shall see below that curvature leads to some striking new results. When $R_{i} \neq \infty$ thick films of gas will still develop at the wall but the thickness will remain finite at $\mu_{c o}^{+}(T)$ and the wall-liquid tension $\gamma_{w l}^{i}\left(R_{i}\right)$ should exhibit the non-analyticities described above. Moreover the density profile $\rho(r)$ is no longer a perfect composite of the two separate interfacial profiles and exhibits interesting features near the wall.

A particular advantage of hard walls is that there is an exact statistical mechanical sumrule [17] which relates the fluid density at contact, $\rho^{i}\left(R_{i}^{+}\right)$, to the pressure $p$ of the bulk (reservoir) fluid and the wall-fluid tension:

$$
k_{B} T \rho^{i}\left(R_{i}^{+}\right)=p+\frac{\alpha_{i} \gamma_{w f}^{i}\left(R_{i}\right)}{R_{i}}+\left(\frac{\partial \gamma_{w f}^{i}\left(R_{i}\right)}{\partial R_{i}}\right)_{T, \mu} .
$$

In the limit $R_{i}=\infty$ the contact density reduces to $p / k_{B} T$, the well-known planar contact theorem. Equation (6) is valid for any one-component fluid at a hard wall. We now insert our result (3) for the tension of the 'dry' interface, $\gamma_{w l}^{i}\left(R_{i}\right)$, into (6) to obtain the contact density $\rho_{\text {liq }}^{i}\left(R_{i}^{+}\right)$at $\mu=\mu_{c o}^{+}(T)$. By subtracting $\rho_{\text {gas }}^{i}\left(R_{i}^{+}\right)$, the contact density when the bulk phase is gas at $\mu=\mu_{c o}^{-}(T)$, we eliminate both the pressure $p\left(\mu_{c o}\right)$ and the (analytic) wall-gas tension obtaining for a hard spherical wall:

$$
k_{B} T\left(\rho_{l i q}^{s}\left(R_{s}^{+}\right)-\rho_{g a s}^{s}\left(R_{s}^{+}\right)\right)=\frac{2 \gamma_{g l}(\infty)}{R_{s}}+\frac{2 \xi \gamma_{g l}(\infty)}{R_{s}^{2}} \ln R_{s}+\text { H.O.T. }
$$




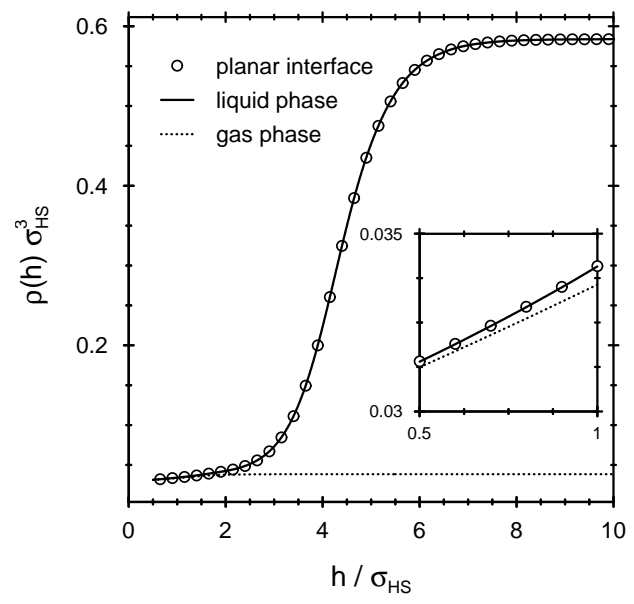

Fig. 1 - The density profile of a square-well fluid adsorbed at a hard wall for $k_{B} T / \varepsilon=1$. Solid curve refers to a liquid at bulk coexistence $\mu_{c o}^{+}(T)$ near a spherical wall of radius $R_{s}=2500 \sigma_{H S}$ and the dotted curve to the gas at $\mu_{c o}^{-}(T)$ at the same wall. Symbols refer to the liquid near a planar wall at chemical potential $\mu-\mu_{c o}(T)=2 \gamma_{g l}(\infty) /\left(R_{s}\left(\rho_{l}-\rho_{g}\right)\right)$ (see text). One observes that the two wall-liquid profiles are indistinguishable in the region of the gas-liquid interface and close to the wall - see inset. Contact occurs at distance $h=\sigma_{H S} / 2$.

and for a hard cylindrical wall:

$$
k_{B} T\left(\rho_{\text {liq }}^{c}\left(R_{c}^{+}\right)-\rho_{g a s}^{c}\left(R_{c}^{+}\right)\right)=\frac{\gamma_{g l}(\infty)}{R_{c}}+\frac{\left(\xi+b_{H}\right) \gamma_{g l}(\infty)}{R_{c}^{2}}+\text { H.O.T. }
$$

where H.O.T refers to terms of higher order in $R_{i}^{-1}$. For a planar wall the contact density is $p\left(\mu_{c o}\right) / k_{B} T$ in both phases so the difference vanishes identically. There are two remarkable features in these results. First the contact density in the liquid phase ('dry' interface) depends on $\gamma_{g l}(\infty)$, the tension of the planar gas-liquid interface, which can be very far from the wall as $R_{i} \rightarrow \infty$ : the first term on the r.h.s. of (7) and (8) is simply the Laplace pressure across the gas-liquid interface. Second the contact density difference for the sphere contains a next to leading order $R_{s}^{-2} \ln R_{s}$ non-analyticity, whereas for the cylinder the next to leading order term is analytic, i.e. $\mathcal{O}\left(R_{c}^{-2}\right)$, despite the fact that both surface tensions are non-analytic at order $R_{i}^{-1} \ln R_{i}$.

In order to test the predictions of the coarse-grained theory we have adopted a fully microscopic DFT approach and performed calculations for a square-well fluid at hard curved walls. The fluid-fluid pair potential $\phi(r)$ is infinite for $r<\sigma_{H S}$, the hard sphere diameter, the width of the well is $0.5 \sigma_{H S}$ and the well-depth is $\varepsilon$. We treat the hard-sphere part of the free-energy functional by means of Rosenfeld's fundamental measure theory [18] and the attractive part using a simple mean-field approximation [6, 16], taking $\phi_{\text {att }}(r)=-\varepsilon$ for $r<$ $1.5 \sigma_{H S}$ and zero otherwise. By minimizing the grand potential functional we determine the equilibrium density profile $\rho(r)$ and the wall-fluid tension for any thermodynamic state; details of the numerical treatment will be given elsewhere. Our DFT approach has the following advantageous features: i) the coexisting densities of gas and liquid, $\rho_{g}$ and $\rho_{l}$, can be calculated precisely, ii) the DFT obeys the sum-rule (6) and the Gibbs adsorption theorem and iii) the key quantities $\xi$ and $\gamma_{g l}(\infty)$ which enter eqs. (3178) can be obtained from independent calculations (for the bulk and for the planar interface) using the same functional. 


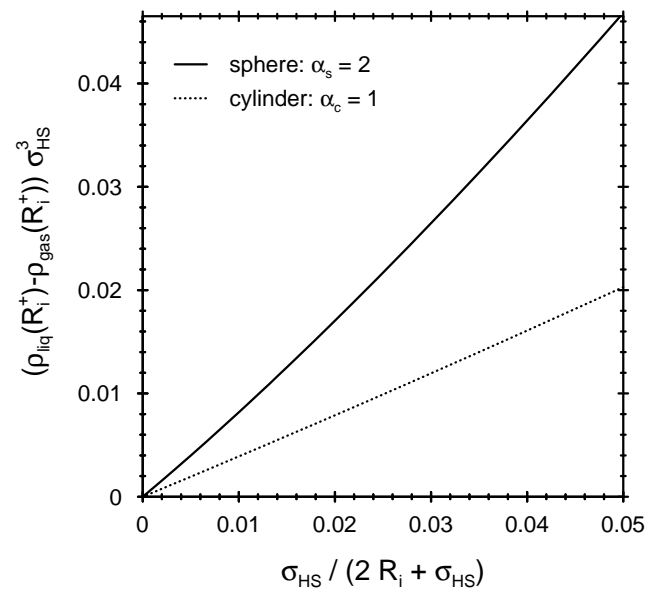

Fig. 2

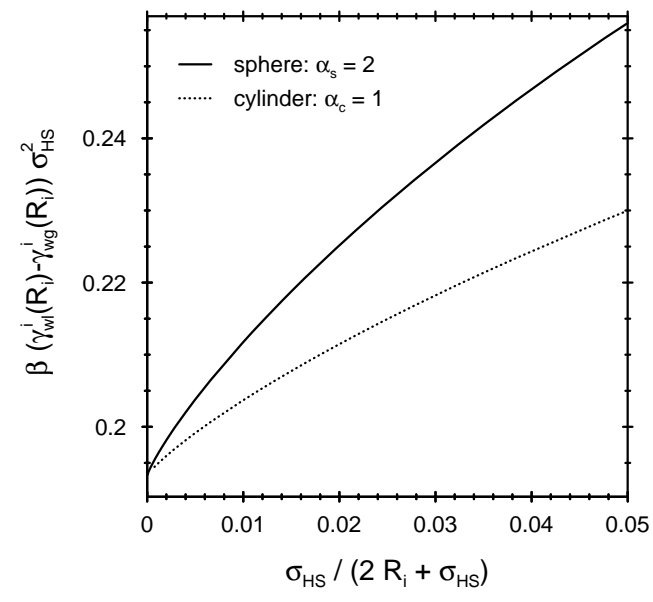

Fig. 3

Fig. 2 - The difference between contact densities of a square-well fluid in the liquid and in the gas phases, at $\mu_{c o}^{ \pm}(T)$, adsorbed at a hard spherical (full line) and hard cylindrical (dotted line) wall of radius $R_{i}$ for $k_{B} T / \varepsilon=1$. From the linear portion of each curve near the origin, we can extract the gas-liquid surface tension $\gamma_{g l}(\infty)$. The next to leading order term is proportional to $R_{s}^{-2} \ln R_{s}$ for the sphere and to $R_{c}^{-2}$ for the cylinder - see eqs. (7) and (8).

Fig. 3 - As in fig. 2 but now for the difference between the surface tensions. For both geometries the leading order curvature dependence is proportional to $\ln R_{i} / R_{i}$ - see eq. (3). In both cases the coefficient is confirmed to be $\alpha_{i} \xi \gamma_{g l}(\infty) / k_{B} T$, with $\xi$ the correlation length in the bulk gas.

We focus first on the density profile. In fig. 1 we display results for $\rho(r)$, at a reduced temperature $k_{B} T / \varepsilon=1$ and chemical potential $\mu_{c o}^{+}(T)$, for the square-well fluid adsorbed on a hard sphere with radius $R_{s}=2500 \sigma_{H S}$. In the same figure we show the profiles for the wall-gas interface at $\mu_{c o}^{-}(T)$ and for the planar interface with $\delta \mu \equiv \mu-\mu_{c o}(T)=2 \gamma_{g l}(\infty) /\left(R_{s}\left(\rho_{l}-\rho_{g}\right)\right)$. One can observe that this translation between bulk field for a planar wall and curvature yields wall-liquid profiles which lie on top of each other. This is a non-trivial result. Although it is evident that within the coarse-grained treatment of eq. (1) the film thickness $l_{e q}^{i}$ is identical for planar and curved walls, there is no reason a priori why the complete microscopic density profiles should be especially close.

In fig. 2 we seek to test the validity of eqs. (7) by examining the difference in the contact densities for the two phases as a function of $R_{i}^{-1}$. Our DFT results are in excellent agreement with the prediction of the coarse grained theory for both spheres and cylinders. In particular we find the data is fit best with a $R_{s}^{-2} \ln R_{s}$ contribution for spheres and without such a contribution for cylinders. It is straightforward to extract the coefficient of the $R_{i}^{-1}$ contribution and we find close agreement, to 1 part in $10^{4}$, with our independent (planar) results for the dimensionless quantity $\alpha_{i} \gamma_{g l}(\infty) \sigma_{H S}^{2} / k_{B} T$.

In fig. 3 we plot the difference between the wall-liquid and wall-gas surface tensions, evaluated at $\mu_{c o}^{ \pm}(T)$, respectively versus $R_{i}^{-1}$ for the square-well fluid adsorbed at spheres and cylinders, again for $k_{B} T / \varepsilon=1$. In accordance with eqs. (3) and (4) we expect that for the sphere the leading order curvature correction to the difference is proportional to $R_{s}^{-1} \ln R_{s}$ with a coefficient given by $2 \xi \gamma_{g l}(\infty)$ where $\xi$ is the correlation length in the bulk gas. For cylinders we employ eqs. (3) and (5) which imply the same type of leading order correction but now with a coefficient $\left(\xi+b_{H}\right) \gamma_{g l}(\infty)$. Our numerical DFT results are completely consistent 
with these predictions with the relevant coefficients agreeing with the planar result to better than $1 \%$. In our calculations we were able to obtain reliable results for $R_{s}$ up to $30000 \sigma_{H S}$ and $R_{c}$ up to $5000 \sigma_{H S}$. For the cylinder our best fit yields a coefficient that has $b_{H}=0$ (or a very small fraction of $\xi$ ) and we conclude that there is no evidence for the $\ln R_{c} / R_{c}$ term conjectured [14] for the cylindrical fluid-fluid interface - see eq. (5). We have confirmed that the same level of agreement between the coarse-grained theory and DFT holds at other temperatures.

Since there is nothing special about the square-well model our predictions for hard spheres and cylinders should be valid for any fluid with short-ranged fluid-fluid potentials that exhibits gas-liquid coexistence and this has important repercussions for the solvation of big solvophobic solute particles. The excess chemical potential of a single hard sphere of radius $R_{s}$ in a solvent is given generally by $\tilde{\mu}^{H S}\left(R_{s}\right)=p 4 \pi R_{s}^{3} / 3+\gamma_{w f}^{s}\left(R_{s}\right) 4 \pi R_{s}^{2}$, where $\gamma_{w f}^{s}$ is the wall-fluid surface tension. Using eq. (3) it follows that the difference between $\tilde{\mu}^{H S}$ evaluated in the liquid, where drying occurs, and in the gas phase, at $\mu_{c o}^{ \pm}(T)$, is

$$
\frac{\left(\tilde{\mu}_{l i q}^{H S}-\tilde{\mu}_{g a s}^{H S}\right)}{4 \pi R_{s}^{2}}=\gamma_{g l}(\infty)\left(1+2 \xi R_{s}^{-1} \ln R_{s}\right)+\text { H.O.T. }
$$

for $R_{s} \rightarrow \infty$. Whilst it is known from recent studies [19] of hard-sphere solutes that $\tilde{\mu}_{\text {liq }}^{H S}$ should contain a gas-liquid surface tension contribution $\gamma_{g l}(\infty) 4 \pi R_{s}^{2}$, the presence of the nonanalytic correction is striking - especially when we recall that the excess chemical potential is the derivative of the excess free energy of the bulk mixture w.r.t. the solute density in the limit of vanishing solute.

The coarse grained treatment that leads to eq. (3) is valid for wetting by either fluid phase. Thus, provided the attractive part of the wall-fluid potential is sufficiently strong and has finite range or decays on a length scale that is shorter than the bulk correlation length $\xi$, now referring to the wetting liquid, adsorption from the saturated gas, at $\mu_{c o}^{-}(T)$, will give rise to a wall-gas tension which has a term $\alpha_{i} \xi \gamma_{g l}(\infty) R_{i}^{-1} \ln R_{i}$. Although the contact theorem (6) is modified when the wall-fluid potential is no longer purely hard the contact density in the presence of a wetting liquid film will still acquire terms which depend on the gas-liquid surface tension, i.e. we expect results equivalent to (7) and (8).

Both the coarse-grained and DFT approaches presented here are mean-field like in that they omit effects of capillary-wave fluctuations in the wetting film [5] 6]. For complete wetting in three dimensions at a planar interface, renormalization group studies based on effective interfacial Hamiltonians with the binding potential (1) find that critical exponents are not altered from their mean-field values but the amplitude of the equilibrium film thickness $l_{e q}(\infty)$ is changed from $\xi$ to $\xi(1+\omega / 2)$, for $\omega<2$, when fluctuations are included [5]. Here $\omega=k_{B} T /\left(4 \pi \gamma_{g l}(\infty) \xi^{2}\right)$ is the usual parameter which measures the strength of capillary-wave fluctuations: $\omega=0$ corresponds to mean-field. We conjecture that our present results for the leading non-analytic term in the surface tension are modified in a similar fashion, i.e. the third term in eq. (3) simply has $\xi$ replaced by $\xi(1+\omega / 2)$. As regards the results (7) and (8) for the difference in the contact densities, the leading-order (Laplace pressure) terms will be unchanged by fluctuations whereas the next to leading order term in eq. (7) will require the same replacement for $\xi$.

For real fluids dispersion forces are always present giving rise to $r^{-6}$ power-law decay of the fluid-fluid pair potential. This leads, in turn, to a wetting film thickness which diverges at coexistence as $l_{e q}\left(R_{i}\right) \sim R_{i}^{1 / 3}$, for $R_{i} \rightarrow \infty$ [10,20]. The present coarse-grained analysis suggests that the non-analyticities in curvature which arise for wetting with such potentials 
will be power laws rather than terms involving logarithms and we are presently investigating these using DFT [21].

We have shown that the isomorphism between bulk field $\delta \mu\left(\rho_{l}-\rho_{g}\right)$ for a planar substrate and Laplace pressure at a curved substrate implied by the binding potential (11) leads to striking consequences for interfacial properties: first one cannot obtain the surface excess free energy (surface tension) of a fluid that wets completely a non-planar substrate by expanding only in powers of the curvature(s). Second the true microscopic density profile near the curved substrate depends on the surface tension of the gas-liquid interface which, for large $R_{i}$, can be far from the substrate. A detailed explanation of this curious behavior will be given elsewhere. Here we merely state that it is associated with the exponential decay (for short-ranged potentials) of the tails of the density profile of the 'free' gas-liquid interface. This is not the first time that analysis of complete wetting or drying at a hard wall has caused surprises and lead to new insight into the fundamental physics of fluid interfaces [15, 22 .

We have benefited from conversations with J.R. Henderson and M. Thomas. R.E. is grateful to S. Dietrich for kind hospitality and to the Humboldt Foundation for support under GRO/1072637 during his stay in Stuttgart.

\section{REFERENCES}

[1] e.g. Dietrich S., in New Approaches to Problems in Liquid State Theory, edited by C. CACCAMO et al. NATO ASI Ser. C, Vol. 529 (Kluwer, Dordrecht) 1999, p. 197.

[2] Bruschi L., Carlin A., and Mistura G., Phys. Rev. Lett., 89 (2002) 166101.

[3] e.g. Rascón C. and Parry A.O., Nature (London), 407 (2000) 986.

[4] Parry A.O., Greenall M.J. and Romero-Enrique J.M., Phys. Rev. Lett., 90 (2003) 046101.

[5] For a general review of wetting see: Dietrich S., in Phase Transitions and Critical Phenomena, edited by C. Domb and J.L. Lebowitz (Academic, London), Vol. 12 1988, p. 1.

[6] For a review of DFT see Evans R.,: in Fundamentals of Inhomogeneous Fluids, edited by D. Henderson (Dekker, New York) 1992, p. 85.

[7] Holyst R. and Poniewierski A., Phys. Rev. B, 36 (1987) 5628. These authors obtained a $R_{s}^{-1} \ln R_{s}$ contribution to the surface tension in a Cahn-like treatment of wetting on a sphere.

[8] Gelfand M.P. and Lipowsky R., Phys. Rev. B, 36 (1987) 8725.

[9] Upton P.J., Indekeu J.O. and Yeomans J.M., Phys. Rev. B, 40 (1989) 666.

[10] Bieker T. and Dietrich S., Physica A, 252 (1998) 85 and references therein.

[11] e.g. Rowlinson J.S., J. Phys.: Condens. Matt., 6 (1994) A1.

[12] DFT studies for hard spheres adsorbed on a hard sphere or cylinder find no contributions to the surface tension of the form $R_{i}^{-1} \ln R_{i}$. Rather they find $\gamma_{w b}^{i}\left(R_{i}\right)=\gamma_{w b}(\infty)\left(1+c_{1} R_{i}^{-1}+c_{2} R_{i}^{-2}+\right.$ H.O.T.), see Bryk, Roth R., Mecke K.R., and Dietrich S., to be published.

[13] Tolman R.C, J. Chem. Phys., 17 (1949) 333.

[14] Henderson J.R. and Rowlinson J.S., J. Phys. Chem., 88 (1984) 6484.

[15] e.g. Henderson J.R. and van Swol F., Mol. Phys., 56 (1985) 1313 and references therein.

[16] Tarazona P. and Evans R., Mol. Phys., 52 (1984) 847; Parry A.O. and Evans R., Mol. Phys., 65 (1988) 455.

[17] Henderson J.R., in Fluid Interfacial Phenomena, edited by C. A. Croxton (Wiley, New York) 1986 , p. 555 - see eq. (12.84a). Our eq. (6) follows by defining $\gamma_{w f}^{i}\left(R_{i}\right)$ as the excess grand potential per unit area.

[18] Rosenfeld Y., Phys. Rev. Lett., 63 (1989) 980.

[19] e.g. Lum K., Chandler D. and Weeks J.D., J. Phys. Chem. B, 103 (1999) 4570; Huang D.M. and Chandler D., Phys. Rev. E, 61 (2000) 1501; Henderson J.R., J. Chem. Phys., 116 (2002) 5039.

[20] Brochard F., J. Chem. Phys., 84 (1986) 4664; Gil T. and Mikeev L.V., Phys. Rev. E, 52 (1995) 772. 
[21] Since the upper critical dimension for complete wetting with power law potentials is $<3$, fluctuations are not important in this case.

[22] Parry A.O. and Evans R., Mol. Phys., 78 (1993) 1527 and references therein. 